\title{
Factor XII as a Risk Marker for Hemorrhagic Stroke: A Prospective Cohort Study
}

\author{
Kristina Johansson $^{\mathrm{a}}$ Jan-Håkan Jansson $^{\mathrm{a}}$ Lars Johansson $^{\mathrm{a}}$ \\ Ingemar Bylesjö $^{b}$ Torbjörn K. Nilsson ${ }^{c}$ Mats Eliasson ${ }^{d}$ \\ Stefan Söderberg ${ }^{\mathrm{e}}$ Marcus Lind ${ }^{\mathrm{a}}$ \\ a Department of Public Health and Clinical Medicine, Skellefteå Research Unit, Umeå \\ University, Umeå, Sweden; ${ }^{b}$ Department of Public Health and Clinical Medicine, Family \\ Medicine, Umeå University, Umeå, Sweden; ' Department of Medical Biosciences/Clinical \\ Chemistry, Umeå University, Umeå, Sweden; ${ }^{\mathrm{d}}$ Department of Public Health and Clinical \\ Medicine, Sunderby Research Unit, Umeå University, Umeå, Sweden; ' Department of Public \\ Health and Clinical Medicine, Umeå University, Umeå, Sweden
}

\section{Keywords}

Coagulation · Biomarkers · Intracranial hemorrhage · Cohort study · Cardiovascular disease

\begin{abstract}
Background: Coagulation factor XII (FXII) is involved in pathological thrombus formation and is a suggested target of anticoagulants. It is unclear whether FXII levels are correlated with cardiovascular risk factors and whether they are associated with myocardial infarction or ischemic or hemorrhagic stroke. The aim of this study was to investigate the correlation between FXII and cardiovascular risk factors in the general population. We also aimed to study the associations between FXII levels and future myocardial infarction and ischemic and hemorrhagic stroke. Methods: This prospective cohort study measured FXII levels in 1,852 randomly selected participants in a health survey performed in northern Sweden in 1994. Participants were followed until myocardial infarction, stroke, death, or until December 31, 2011. Results: During the median follow-up of 17.9 years, 165 individuals were diagnosed with myocardial infarction, 108 with ischemic stroke, and 30 with hemorrhagic stroke. There were weak correlations between FXII and body mass index, cholesterol, and hypertension. There was no association between FXII and myocardial infarction or ischemic stroke, neither in univariable Cox regression analysis nor after adjustment for age, sex, smoking, body mass index, cholesterol, hypertension, and diabetes. In univariable Cox regression analysis, the hazard ratio for the association between FXII levels and hemorrhagic stroke was 1.42 per SD
\end{abstract}


(95\% confidence interval: $0.99-2.05)$. In the multivariable model, higher levels of FXII were associated with increased risk of hemorrhagic stroke (hazard ratio 1.51 per SD; 95\% confidence interval: 1.03-2.21). Conclusion: We found an independent association between FXII levels and the risk of hemorrhagic stroke, but not between FXII levels and ischemic stroke or myocardial infarction.

\section{Introduction}

Myocardial infarction and stroke are leading causes of death worldwide [1]. There are biochemical markers that can be used to identify individuals with a high risk of cardiovascular events [2], and it has been suggested that factor XII (FXII), a protein involved in the intrinsic pathway of blood coagulation, could be a risk marker for thromboembolic disease [3-7]. Recent animal studies have suggested that FXII may have an important role in pathological thrombus formation [8-11] and that it is affected by the inflammatory process in acute stroke [12]. FXII inhibitors have been suggested as promising new antithrombotic agents [13], while epidemiological studies have reported that low levels of FXII are not associated with an increased risk of bleeding $[13,14]$. In addition, animals treated with FXII inhibitors do not exhibit an increased bleeding risk, indicating that anticoagulants targeting FXII might be a safe alternative to current anticoagulant treatments [8-11]. Earlier research that investigated FXII as a possible risk marker for myocardial infarction and stroke reported contradicting results and rarely looked at data from the general population [3-7].

It is important to identify correlations between FXII levels and traditional cardiovascular risk factors in order to account for confounding factors, and previous studies have shown contradicting results with weak positive or absent associations with classical cardiovascular risk factors $[15,16]$.

FXII levels decrease in acute phase reactions [17]. Consequently, interpreting FXII levels in acute conditions is difficult, and prospective studies are needed to investigate FXII as a possible risk marker for disease. The American Atherosclerosis Risk in Communities (ARIC) study has examined the association between FXII levels and the risk of ischemic stroke and myocardial infarction and found no associations between FXII levels and future myocardial infarction or ischemic stroke. However, only people aged 45-64 years were included in the cohort $[18,19]$. No studies have examined the relationship between FXII levels and hemorrhagic stroke, although sporadic case reports have linked FXII deficiency to intracranial bleeding $[20,21]$. Ischemic and hemorrhagic stroke share many risk factors and it has been suggested that these conditions are two different manifestations of common underlying pathogenetic mechanisms [22]. The second Northwick Park Study (NPHS-II) found that lower levels of activated FXII-inhibitory complex seem to increase the risk of developing coronary heart disease and ischemic stroke in healthy middle-aged men [23].

Since the correlation between FXII and cardiovascular risk factors and the associations between FXII and future cardiovascular disease remain unclear, we investigated these relationships in a prospective population-based cohort study that evaluated the presence of cardiovascular risk factors in participants at baseline and involved 18 years of follow-up. The aim of this study was to investigate the correlation between FXII and cardiovascular risk factors and the associations between FXII levels and future myocardial infarction and ischemic and hemorrhagic stroke in the general population. 


\section{Methods}

This prospective cohort study included the participants of a health survey that was conducted in northern Sweden in 1994. The participants were followed until myocardial infarction, stroke, death, or until the end of the study on December 31, 2011, whichever came first.

\section{Study Population and Setting}

The study population included participants in the 1994 Monitoring Trends and Determinants in Cardiovascular Disease (MONICA) project in northern Sweden, a health survey initiated by the World Health Organization (WHO) with the aim of studying risk factors and trends in cardiovascular disease [24]. An age-stratified sample of 2,500 individuals aged 25-74 years, 1,250 women and 1,250 men, were invited to participate. Individuals were randomly selected among the residents of the counties of Västerbotten and Norrbotten, the two northernmost counties of Sweden, which had a combined population of 527,423 in 1994 [25]. Of the 2,500 people who were invited to participate, 1,921 attended the health survey and of these, 1,852 had a valid FXII measurement and were followed as a cohort until December 31,2011 . These 1,852 people comprised the study population. Information about dates of death was obtained from the population register at Statistics Sweden.

\section{Event Registration}

The National Patient Register and the Swedish Cause of Death Register were searched for ICD-9 and ICD-10 diagnosis codes for myocardial infarction (410, I21), ischemic stroke (434, I63), and hemorrhagic stroke $(430,431$, I60, I61) for the years 1994 through 2011. Ten individuals with unspecified stroke $(436,164)$ were excluded from analyses concerning ischemic or hemorrhagic stroke. A participant flowchart is shown in Figure 1.

\section{Measurements and Definitions}

The baseline health survey was performed between January 1 and April 22, 1994, by dedicated teams that examined the participants at the nearest primary health care center. Participants underwent a health examination with blood samples and were asked to complete a questionnaire.

Data on smoking habits and antihypertensive and antidiabetic treatment were collected by a questionnaire. Smoking was defined as self-reported current or former smoking of at least 1 cigarette/day. Weight was recorded to the nearest $0.2 \mathrm{~kg}$ using a balance scale that was calibrated daily. Participants wore light clothing and no shoes while being weighed. Height without shoes was measured to the nearest centimeter. Body mass index (BMI) was calculated as weight in kilograms divided by the square of height in meters.

Blood pressure was measured twice using a Hawksley random zero sphygmomanometer with the participant in a sitting position after resting for $5 \mathrm{~min}$. The mean of the 2 measurements was recorded. Hypertension was defined as systolic blood pressure $\geq 140 \mathrm{~mm} \mathrm{Hg}$ or diastolic blood pressure $\geq 90 \mathrm{~mm} \mathrm{Hg}$ or the use of antihypertensive drugs during the 2 weeks preceding the health survey.

Blood samples were retrieved after a minimum of $4 \mathrm{~h}$ of fasting. Total serum cholesterol was analyzed using an enzymatic method (BM Monotest Cholesterol CHOD-PAP, Boehringer Mannheim GmbH, Mannheim, Germany). Venous blood samples for measuring fasting plasma glucose and 2-h oral glucose tolerance test (OGTT) plasma glucose were drawn into sodium fluoride tubes and analyzed using a hexokinase method (Boehringer Mannheim Automated Analysis for BM/Hitachi System 717, Mannheim, Germany). Diabetes was defined as fasting plasma glucose $\geq 7.0 \mathrm{mmol} / \mathrm{L}, 2$-h OGTT plasma glucose $\geq 11.1 \mathrm{mmol} / \mathrm{L}$ or self-reported current use of antidiabetic medication. 


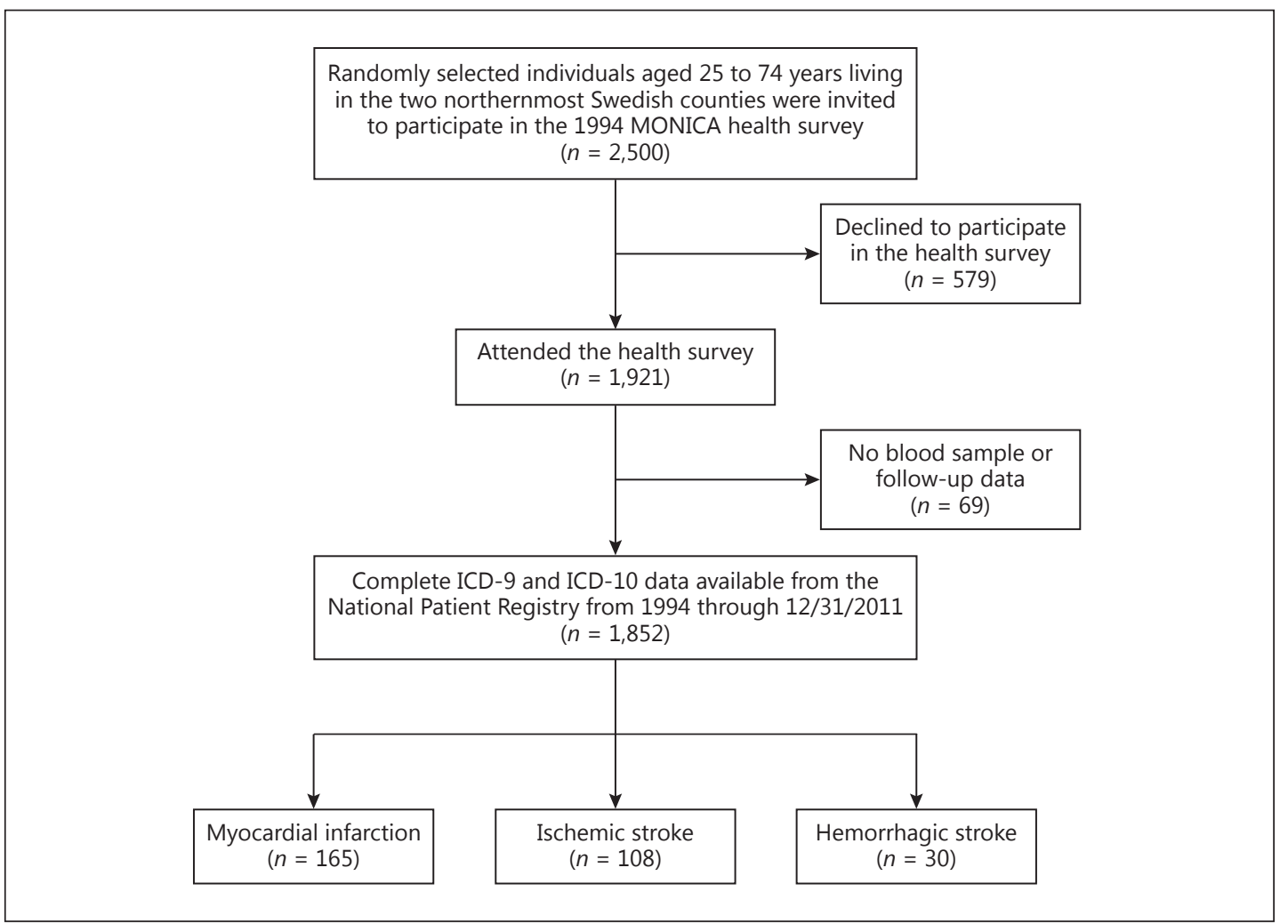

Fig. 1. Participant flow diagram.

Blood samples were collected for future research. Erythrocytes and plasma were separated by centrifugation at $1,500 \mathrm{~g}$ for $15 \mathrm{~min}$, and plasma was divided into aliquots and stored at $-80^{\circ} \mathrm{C}$ until analysis. For FXII analysis, blood was collected in EDTA Venoject tubes (Terumo, Leuven, Belgium). Zymogen FXII levels were determined using a chromogenic substrate assay described by Tankersley et al. [26] which is based on dextran sulfate-induced activation of prekallikrein to kallikrein by activated FXII. The rate of kallikrein generation over time, mediated by activated FXII, is recorded by a chromogenic substrate sensitive to kallikrein, S-2302, and is proportional to the original FXII zymogen concentration in the participant's plasma sample. Reagents were prepared as described by Tankersley et al. [26] except that the prekallikrein purification was scaled up to allow the processing of $1.5 \mathrm{~L}$ of human plasma and the analyses were performed on a Hitachi 911 automatic analyzer. Pooled plasma from 20 healthy individuals (laboratory staff) was used to construct standard curves, and calibration to international units per milliliter was performed using a WHO calibrator plasma. All samples were analyzed at the same location and at the same time (1997). Laboratory staff had no knowledge of event status.

\section{Missing Values}

The date of the health survey was missing in 4 participants, who were assigned the median date of the health survey. Questionnaire data on smoking habits, antihypertensive treatment, and antidiabetic treatment were missing in 4, 6, and 6 participants, respectively. Of the 6 patients with missing antidiabetic treatment data, 2 had valid OGTT results. BMI data were missing in 4 participants. Blood pressure measurements were missing in 1 participant. None of the participants had missing cholesterol levels data. Participants with a missing value for one variable were excluded from all analyses that included that variable. 


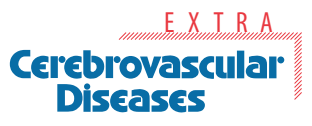

Table 1. Baseline characteristics of the study population $(n=1,852)$

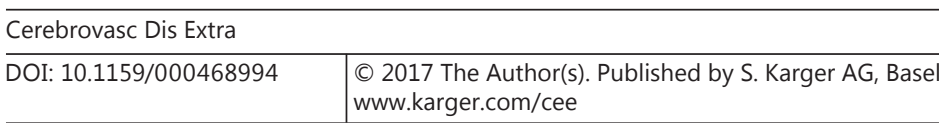

Johansson et al.: Factor XII and Risk for Hemorrhagic Stroke
Characteristics

Age, years

Female, $n$

Smokera, $n$

Median BMI, kg/m²

Median cholesterol, $\mathrm{mmol} / \mathrm{L}$

Hypertension $^{\mathrm{b}}, n$

Diabetes ${ }^{\mathrm{c}}, n$

Median factor XII, IU/mL

\author{
$50(38-62)$ \\ $938(50.6)$ \\ $923(49.9)$ \\ $25.5(23.1-28.3)$ \\ $6.0(5.2-6.9)$ \\ $710(38.4)$ \\ $84(4.5)$ \\ $1.12(0.92-1.32)$
}

Values in parentheses are 1st-3rd quartiles or percentages. BMI, body mass index. ${ }^{a}$ Current or former smoker. ${ }^{b}$ Systolic blood pressure $\geq 140 \mathrm{~mm} \mathrm{Hg}$, diastolic blood pressure $\geq 90 \mathrm{~mm} \mathrm{Hg}$, or antihypertensive treatment. ${ }^{\mathrm{c}}$ Pharmacologically treated diabetes, fasting plasma glucose $\geq 7.0 \mathrm{mmol} / \mathrm{L}$, or 2 -h oral glucose tolerance test plasma glucose $\geq 11.1$ $\mathrm{mmol} / \mathrm{L}$.

\section{Statistical Methods}

The number of observations, percentages, and the median and interquartile range were calculated for the baseline characteristics. The Spearman rank order correlation was used for testing the correlation between FXII levels and traditional cardiovascular risk factors. A twosided $p$ value of $<0.05$ was considered significant. The normality of distribution was tested by visual inspection of $Q-Q$ plots. All variables were considered normally distributed. The assumption of proportional hazards was verified graphically using Kaplan-Meier survival curves. Myocardial infarction and ischemic and hemorrhagic stroke were analyzed separately, and only the first occurrence of each outcome during the study period was considered. The time-to-event was defined as the time from the health survey date to the diagnosis date.

Cox proportional hazard regression was used to investigate the associations between FXII levels, traditional cardiovascular risk factors, and the outcomes in univariable and multivariable analyses. All variables included in the univariable analyses were included in the multivariable model. In order to facilitate comparisons between hazard ratios (HRs), all continuous variables were divided by their standard deviations (SDs) before being entered in the model. FXII was also analyzed as a categorical variable using tertiles to enable the detection of a stepwise change in risk. All calculations were performed with SPSS software version 22.0 (Chicago, IL, USA).

The study was approved by the Regional Ethics Review Board, Umeå, Sweden. All study participants provided informed written consent.

\section{Results}

The baseline characteristics of the 1,852 participants are shown in Table 1. The median participant age was 50 years, and $51 \%$ were women. The median duration of follow-up was 17.9 years, with a total follow-up time of 30,372 patient-years. The median FXII level was 1.12 IU/mL. Correlations between FXII levels and age, sex, smoking, BMI, cholesterol, hypertension, and diabetes are shown in Table 2. High FXII levels were correlated with high BMI, high cholesterol levels, and hypertension. The associations between FXII levels and incident myocardial infarction, ischemic stroke, and hemorrhagic stroke are shown in Table 3 and in Figure 2. 
Table 2. Spearman correlation coefficients between factor XII and cardiovascular risk factors

\begin{tabular}{llllllll}
\hline & Age & Sex & Smoking $^{\mathrm{a}}$ & BMI & Cholesterol & Hypertension $^{\mathrm{b}}$ & Diabetes $^{\mathrm{c}}$ \\
\hline Factor XII & -0.02 & $<0.01$ & -0.03 & $0.07^{* *}$ & $0.13^{* *}$ & $0.06^{*}$ & 0.04 \\
\hline
\end{tabular}

BMI, body mass index. ${ }^{*} p<0.05 ;{ }^{* *} p<0.001{ }^{\text {a }}$ Current or former smoker. ${ }^{\mathrm{b}}$ Systolic blood pressure $\geq 140$ $\mathrm{mm} \mathrm{Hg}$, diastolic blood pressure $\geq 90 \mathrm{~mm} \mathrm{Hg}$, or antihypertensive treatment. ${ }^{\mathrm{c}}$ Pharmacologically treated diabetes, fasting plasma glucose $\geq 7.0 \mathrm{mmol} / \mathrm{L}$, or 2 -h oral glucose tolerance test plasma glucose $\geq 11.1$ $\mathrm{mmol} / \mathrm{L}$.

Table 3. Hazard ratios with $95 \%$ confidence intervals showing the associations between cardiovascular risk factors, factor XII, and myocardial infarction, ischemic and hemorrhagic stroke

\begin{tabular}{|c|c|c|c|c|c|c|}
\hline & \multicolumn{2}{|c|}{ Myocardial infarction $(n=165)$} & \multicolumn{2}{|c|}{ Ischemic stroke $(n=108)$} & \multicolumn{2}{|c|}{ Hemorrhagic stroke $(n=30)$} \\
\hline & univariable & multivariable & univariable & multivariable & univariable & multivariable \\
\hline $\begin{array}{l}\text { Age by } 10 \text {-year } \\
\text { groups }\end{array}$ & $2.27(1.96-2.63)^{*}$ & $2.06(1.75-2.43)^{*}$ & $2.97(2.41-3.65)^{*}$ & $2.76(2.20-3.47)^{*}$ & $1.76(1.30-2.37)^{*}$ & $1.79(1.25-2.56)^{*}$ \\
\hline Sex/male & $2.37(1.71-3.29)^{*}$ & $2.54(1.82-3.56)^{*}$ & $1.60(1.09-2.35)^{*}$ & $1.54(1.04-2.29)^{*}$ & $0.95(0.46-1.95)$ & $0.87(0.42-1.81)$ \\
\hline Smokinga & $1.39(1.02-1.90)^{*}$ & $1.45(1.06-1.98)^{*}$ & $1.09(0.75-1.59)$ & $1.26(0.85-1.85)$ & $1.81(0.86-3.80)$ & $2.07(0.97-4.39)$ \\
\hline BMI per SD & $1.39(1.23-1.57)^{*}$ & $1.17(1.00-1.37)^{*}$ & $1.25(1.06-1.48)^{*}$ & $0.99(0.81-1.21)$ & $1.14(0.82-1.60)$ & $0.95(0.65-1.37)$ \\
\hline Cholesterol per SD & $1.62(1.41-1.86)^{*}$ & $1.28(1.09-1.51)^{*}$ & $1.33(1.11-1.59)^{*}$ & $0.91(0.73-1.12)$ & $1.16(0.82-1.64)$ & $0.81(0.54-1.21)$ \\
\hline Hypertension $^{\mathrm{b}}$ & $3.63(2.63-5.01)^{*}$ & $1.35(0.95-1.94)$ & $4.95(3.25-7.54)^{*}$ & $1.90(1.20-3.02)^{*}$ & $2.72(1.31-5.65)^{*}$ & $1.51(0.65-3.54)$ \\
\hline Diabetes $^{c}$ & $3.70(2.32-5.90)^{*}$ & $1.86(1.15-3.02)^{*}$ & $3.01(1.61-5.62)^{*}$ & $1.26(0.67-2.39)$ & $2.79(0.84-9.19)$ & $1.46(0.43-5.01)$ \\
\hline FXII per SD & $1.05(0.90-1.22)$ & $1.04(0.88-1.23)$ & $0.92(0.77-1.11)$ & $0.95(0.78-1.16)$ & $1.42(0.99-2.05)$ & $1.51(1.03-2.21)^{*}$ \\
\hline
\end{tabular}

Associations are presented as univariable and multivariable hazard ratios. SD, standard deviation; BMI, body mass index; FXII, factor XII. * $p<0.05$.

${ }^{a}$ Current or former smoker. ${ }^{b}$ Systolic blood pressure $\geq 140 \mathrm{~mm} \mathrm{Hg}$, diastolic blood pressure $\geq 90 \mathrm{~mm}$ Hg, or antihypertensive treatment. ${ }^{\mathrm{c}}$ Pharmacologically treated diabetes, fasting plasma glucose $\geq 7.0 \mathrm{mmol} / \mathrm{l}$, or 2 -h oral glucose tolerance test plasma glucose $\geq 11.1 \mathrm{mmol} / \mathrm{l}$.

Fig. 2. Associations between levels of factor XII per standard deviation and risk of myocardial infarction, ischemic stroke, and hemorrhagic stroke. Associations are presented as hazard ratios with 95\% confidence intervals. Results from univariable analyses are presented as dashed lines and results from multivariable analyses are presented as solid lines.

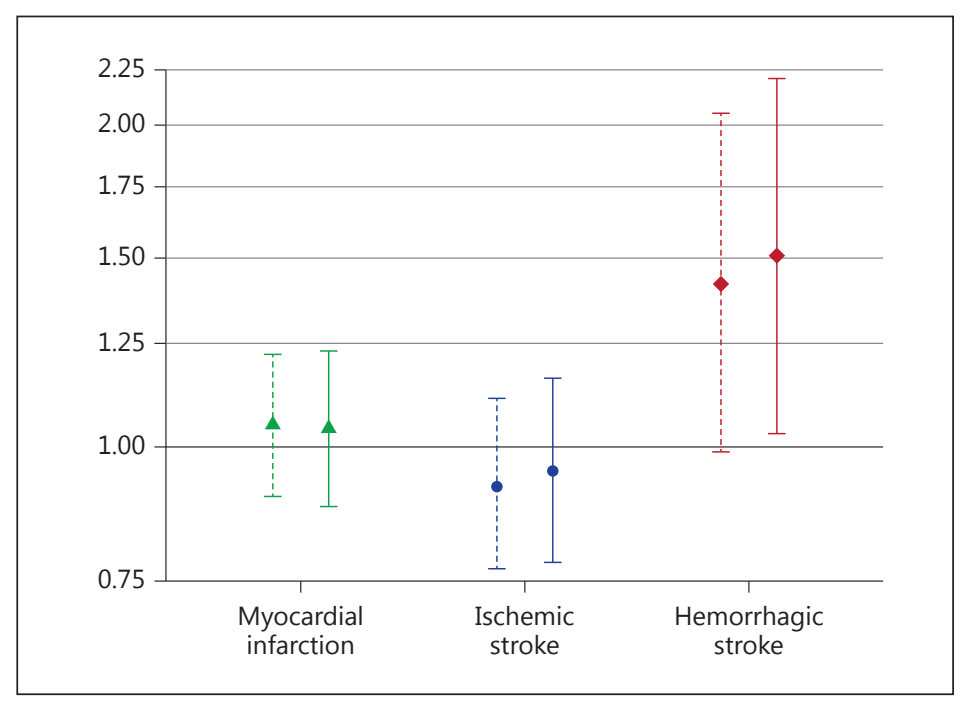




\section{Myocardial Infarction}

During follow-up, 165 individuals were diagnosed with myocardial infarction after a median time-to-event of 9.5 years. Univariable Cox proportional hazard regression analysis showed no association between FXII levels and myocardial infarction (HR 1.05 per SD; 95\% CI: $0.90-1.22$ ). There was no association between tertiles of FXII and myocardial infarction (data not shown). A multivariable Cox regression model adjusted for age, sex, smoking, BMI, cholesterol, hypertension, and diabetes showed no association between FXII levels and myocardial infarction (HR 1.04 per SD; 95\% CI: 0.88-1.23).

\section{Ischemic Stroke}

During the study period, 108 individuals were diagnosed with ischemic stroke after a median time-to-event of 11.4 years. Univariable Cox proportional hazard regression analysis showed no association between FXII levels and ischemic stroke (HR 0.92 per SD; 95\% CI: 0.77-1.11). There was no association between tertiles of FXII and ischemic stroke (data not shown). In a multivariable Cox regression model adjusted for age, sex, smoking, BMI, cholesterol, hypertension, and diabetes, there was no association between FXII levels and ischemic stroke (HR 0.95 per SD; 95\% CI: 0.78-1.16).

\section{Hemorrhagic Stroke}

During the study period, 30 individuals were diagnosed with hemorrhagic stroke after a median time-to-event of 11.1 years. Of these individuals, 20 had intracerebral bleeding and 10 had subarachnoid bleeding. In univariable Cox regression analysis, the HR for the association between FXII levels and hemorrhagic stroke was 1.42 per SD (95\% CI: 0.99-2.05). In a multivariable model adjusted for age, sex, smoking, BMI, cholesterol, hypertension, and diabetes, higher levels of FXII were significantly associated with increased risk of hemorrhagic stroke (HR 1.51 per SD; 95\% CI: 1.03-2.21). When the relationship between tertiles of FXII and hemorrhagic stroke was investigated using univariable Cox regression, the HR was 2.10 (95\% CI: 0.79-5.59) for the 2nd tertile and 2.00 (95\% CI: 0.75-5.33) for the 3rd tertile using the lowest tertile of FXII as the reference category. When analyzing intracerebral bleeding and subarachnoid bleeding separately, the adjusted HR for the association between FXII and intracerebral bleeding was 1.50 per SD of FXII (95\% CI: $0.95-2.34)$, and for subarachnoid bleeding, the HR was 1.30 per SD of FXII (95\% CI: 0.69-2.44).

\section{Discussion}

This prospective cohort study found a significant association between baseline FXII levels and the risk of hemorrhagic stroke in a model adjusted for age, sex, smoking, BMI, cholesterol, hypertension, and diabetes. In contrast, FXII levels did not predict myocardial infarction or ischemic stroke. There were weak (but in a few factors significant) correlations between FXII levels and cardiovascular risk factors in our study, which is in line with previous findings $[18,27]$.

In our study, there was no association between FXII levels and myocardial infarction or ischemic stroke. The prospective ARIC study found no significant differences in FXII levels between individuals with and without cardiovascular disease $[18,19]$. A case-control study of young women with ischemic stroke and healthy controls found no differences in FXII levels between cases and controls [28].

In our study, we found an association between high FXII levels and increased risk of hemorrhagic stroke. Both epidemiological studies and animal studies have demonstrated that low FXII levels are not associated with an increased risk of bleeding $[9,14]$. An associ- 
ation between high FXII levels and increased risk of hemorrhagic stroke or other bleeding conditions has not been described previously. While there have been observational studies of the putative association between FXII levels and thromboembolic disease, to our knowledge there have been no prospective population-based studies that investigated FXII levels as a possible risk marker for hemorrhagic stroke. However, decreased levels of other coagulation factors, such as von Willebrand factor, are associated with future hemorrhagic stroke [29]. Both intracerebral hemorrhage and subarachnoid bleeding are conditions with a high mortality [30, 31]. Using a biomarker such as FXII to find high-risk groups in which preventive strategies could be implemented could help lower the number of deaths attributable to hemorrhagic stroke.

Hypertension is the most important risk factor for intracerebral hemorrhage. Chronic hypertension induces injuries and inflammation in the arterial wall which over time make the vessel fragile. Ultimately, the vessel may rupture and intracerebral hemorrhage ensues [32, 33]. The association we found between high FXII levels and hemorrhagic stroke may reflect the presence of a vessel wall injury in the cerebral arteries making them prone to rupture rather than occlusion. Since the median follow-up time in our study was 18 years, elevated levels of FXII could reflect a low-grade vessel wall injury over long time.

\section{Strengths}

The strengths of this study include its population-based study design, the inclusion of a random sample of the population with a wide range of ages (25-74 years), and the long follow-up period (median follow-up time of 18 years). The participation rate in the Northern Sweden MONICA survey was $80 \%$ in 1994. Telephone interviews of nonparticipants were conducted to assess possible nonrespondent bias in the MONICA Northern Sweden survey, and the overall nonrespondent bias was low [34]. Therefore, we consider the study population to be a representative sample of the population in northern Sweden. With 1,852 participants, it is unlikely that we missed a clinically relevant correlation between FXII and cardiovascular risk factors. The myocardial infarction and stroke diagnoses were collected from the National Patient Register. The validity of National Patient Register diagnoses of stroke and myocardial infarction, which were used as outcomes in this study, is high $[35,36]$.

\section{Limitations}

As there were a limited number of events, especially for hemorrhagic stroke, the results of our study should be considered as hypothesis generating. Due to the limited number of bleeding events, we evaluated intracerebral and subarachnoid bleeding as a combined eventhemorrhagic stroke. Our results indicate an association between FXII levels and both intracerebral bleeding and subarachnoid bleeding. As the pathophysiology of these diseases differs, forthcoming studies should investigate the association between FXII levels and intracerebral and subarachnoid bleeding separately. Due to the limited number of events, it is possible that we failed to detect weak associations between FXII levels, myocardial infarction and ischemic stroke. However, this seems unlikely since the HRs for the associations between FXII levels, myocardial infarction and ischemic stroke were very close to 1.0. It would also have been interesting to analyze different subtypes of ischemic stroke separately, as other studies have shown that levels of other biomarkers differ across subtypes [37-39]. Another limitation is the inclusion of inhabitants from just one geographical area. Notably, the population levels of FXII vary between countries and ethnicities, perhaps due to the strong association between FXII levels and FXII genetic polymorphisms [40, 41]. FXII levels were measured at a single time point, which may have led to an underestimation of the association between the risk marker and the outcome [42]. However, a previous study showed good within-person correlation of FXII antigen levels measured at baseline and 5 years later [14]. 
In conclusion, there were no relevant correlations between FXII levels and cardiovascular risk factors. There were no associations between FXII levels and the risk of myocardial infarction or ischemic stroke. FXII levels predicted hemorrhagic stroke in a model that was adjusted for sex, age, and traditional cardiovascular risk factors. However, before suggesting high FXII as a novel risk marker for hemorrhagic stroke, further studies are needed to verify this association.

\section{Acknowledgements}

This work was supported by grants from the Västerbotten County Council and from the Foundation for Medical Research in Skellefteå. The MONICA Study was supported by the Västerbotten and Norrbotten County Councils.

\section{Author Contributions}

K. Johansson, M. Lind, L. Johansson, and J.-H. Jansson were responsible for the study concept and design. I. Bylesjö proposed the study of FXII levels in this cohort and took part in the planning of the study. T.K. Nilsson prepared the reagent and performed the FXII assays. $\mathrm{K}$. Johansson was responsible for statistical analysis, interpretation of the data, and drafting of the manuscript. M. Lind, L. Johansson, J.-H. Jansson, T.K. Nilsson, M. Eliasson, and S. Söderberg were responsible for critical revision of the intellectual content of the manuscript. M. Eliasson was responsible for clinical data acquisition and blood sampling. S. Söderberg was responsible for event registration. All authors approved the final version of the manuscript.

\section{References}

1 Mozaffarian D, Benjamin EJ, Go AS, Arnett DK, Blaha MJ, Cushman M, de Ferranti S, Després J-P, Fullerton HJ, Howard VJ, Huffman MD, Judd SE, Kissela BM, Lackland DT, Lichtman JH, Lisabeth LD, Liu S, Mackey RH, Matchar DB, McGuire DK, et al: Heart disease and stroke statistics - 2015 update: a report from the American Heart Association. Circulation 2015;131:e29-e322.

-2 Vasan RS: Biomarkers of cardiovascular disease: molecular basis and practical considerations. Circulation 2006;113:2335-2362.

-3 Cushman M, O'Meara ES, Folsom AR, Heckbert SR: Coagulation factors IX through XIII and the risk of future venous thrombosis: the Longitudinal Investigation of Thromboembolism Etiology. Blood 2009;114:28782883.

4 Gallimore MJ, Harris SL, Jones DW, Winter M: Plasma levels of factor XII, prekallikrein and high molecular weight kininogen in normal blood donors and patients having suffered venous thrombosis. Thromb Res 2004;114:91-96.

5 Bach J, Endler G, Winkelmann BR, Boehm BO, Maerz W, Mannhalter C, Hellstern P: Coagulation factor XII (FXII) activity, activated FXII, distribution of FXII C46T gene polymorphism and coronary risk. J Thromb Haemost 2008;6:291-296.

-6 Zito F, Lowe GD, Rumley A, McMahon AD, Humphries SE: Association of the factor XII 46C >T polymorphism with risk of coronary heart disease (CHD) in the WOSCOPS study. Atherosclerosis 2002;165:153-158.

7 Santamaria A, Mateo J, Tirado I, Oliver A, Belvis R, Marti-Fabregas J, Felices R, Soria JM, Souto JC, Fontcuberta $\mathrm{J}$ : Homozygosity of the $\mathrm{T}$ allele of the $46 \mathrm{C}$-> T polymorphism in the $\mathrm{F} 12$ gene is a risk factor for ischemic stroke in the Spanish population. Stroke 2004;35:1795-1799.

-8 Matafonov A, Leung PY, Gailani AE, Grach SL, Puy C, Cheng Q, Sun MF, McCarty OJ, Tucker EI, Kataoka H, Renne T, Morrissey JH, Gruber A, Gailani D: Factor XII inhibition reduces thrombus formation in a primate thrombosis model. Blood 2014;123:1739-1746.

-9 Renne T, Pozgajova M, Gruner S, Schuh K, Pauer HU, Burfeind P, Gailani D, Nieswandt B: Defective thrombus formation in mice lacking coagulation factor XII. J Exp Med 2005;202:271-281. 
10 Larsson M, Rayzman V, Nolte MW, Nickel KF, Bjorkqvist J, Jamsa A, Hardy MP, Fries M, Schmidbauer S, Hedenqvist P, Broome M, Pragst I, Dickneite G, Wilson MJ, Nash AD, Panousis C, Renne T: A factor XIIa inhibitory antibody provides thromboprotection in extracorporeal circulation without increasing bleeding risk. Sci Transl Med 2014;6:222ra17.

-11 Chen JW, Figueiredo JL, Wojtkiewicz GR, Siegel C, Iwamoto Y, Kim DE, Nolte MW, Dickneite G, Weissleder R, Nahrendorf M: Selective factor XIIa inhibition attenuates silent brain ischemia: application of molecular imaging targeting coagulation pathway. JACC Cardiovasc Imaging 2012;5:1127-1138.

12 Nieswandt B, Kleinschnitz C, Stoll G: Ischaemic stroke: a thrombo-inflammatory disease? J Physiol 2011;589: 4115-4123.

13 Kenne E, Renne T: Factor XII: a drug target for safe interference with thrombosis and inflammation. Drug Discov Today 2014;19:1459-1464.

14 Zeerleder S, Schloesser M, Redondo M, Wuillemin WA, Engel W, Furlan M, Lammle B: Reevaluation of the incidence of thromboembolic complications in congenital factor XII deficiency - a study on 73 subjects from 14 Swiss families. Thromb Haemost 1999;82:1240-1246.

-15 Miller GJ, Esnouf MP, Burgess AI, Cooper JA, Mitchell JP: Risk of coronary heart disease and activation of factor XII in middle-aged men. Arterioscler Thromb Vasc Biol 1997;17:2103-2106.

$\checkmark 16$ Luxembourg B, Schmitt J, Humpich M, Glowatzki M, Seifried E, Lindhoff-Last E: Intrinsic clotting factors in dependency of age, sex, body mass index, and oral contraceptives: definition and risk of elevated clotting factor levels. Blood Coagul Fibrinolysis 2009;20:524-534.

-17 Citarella F, Felici A, Brouwer M, Wagstaff J, Fantoni A, Hack CE: Interleukin-6 downregulates factor XII production by human hepatoma cell line (HepG2). Blood 1997;90:1501-1507.

18 Yamagishi K, Aleksic N, Hannan PJ, Folsom AR: Coagulation factors II, V, IX, X, XI, and XII, plasminogen, and alpha-2 antiplasmin and risk of coronary heart disease. J Atheroscler Thromb 2010;17:402-409.

-19 Suri MF, Yamagishi K, Aleksic N, Hannan PJ, Folsom AR: Novel hemostatic factor levels and risk of ischemic stroke: the Atherosclerosis Risk in Communities (ARIC) Study. Cerebrovasc Dis 2010;29:497-502.

20 Kaspera W, Rothkegel K, Stadnicki A: Subdural hematoma in a patient with Hageman trait - case report. Neurol Med Chir (Tokyo) 2005;45:640-644.

-21 Kovalainen S, Myllyla VV, Tolonen U, Hokkanen E: Recurrent subarachnoid haemorrhages in patient with Hageman factor deficiency. Lancet 1979;1:1035-1036.

22 Johansson L, Jansson JH, Boman K, Nilsson TK, Stegmayr B, Hallmans G: Tissue plasminogen activator, plasminogen activator inhibitor-1, and tissue plasminogen activator/plasminogen activator inhibitor-1 complex as risk factors for the development of a first stroke. Stroke 2000;31:26-32.

-23 Govers-Riemslag JW, Smid M, Cooper JA, Bauer KA, Rosenberg RD, Hack CE, Hamulyak K, Spronk HM, Miller GJ, ten Cate H: The plasma kallikrein-kinin system and risk of cardiovascular disease in men. J Thromb Haemost 2007;5:1896-1903.

24 Stegmayr B, Lundberg V, Asplund K: The events registration and survey procedures in the Northern Sweden MONICA Project. Scand J Public Health Suppl 2003;61:9-17.

25 Population Statistics, Statistics Sweden. http://www.scb.se/en_/Finding-statistics/Statistics-by-subjectarea/Population/ (accessed October 2, 2016).

-26 Tankersley DL, Alving BM, Finlayson JS: Activation of factor XII by dextran sulfate: the basis for an assay of factor XII. Blood 1983;62:448-456.

27 Endler G, Marsik C, Jilma B, Schickbauer T, Quehenberger P, Mannhalter C: Evidence of a U-shaped association between factor XII activity and overall survival. J Thromb Haemost 2007;5:1143-1148.

-28 Siegerink B, Rosendaal FR, Algra A: Antigen levels of coagulation factor XII, coagulation factor XI and prekallikrein, and the risk of myocardial infarction and ischemic stroke in young women. J Thromb Haemost 2014;12: 606-613.

-29 Johansson L, Jansson JH, Stegmayr B, Nilsson TK, Hallmans G, Boman K: Hemostatic factors as risk markers for intracerebral hemorrhage: a prospective incident case-referent study. Stroke 2004;35:826-830.

-30 Carlsson M, Wilsgaard T, Johnsen SH, Vangen-Lønne AM, Løchen ML, Njølstad I, Mathiesen EB: Temporal trends in incidence and case fatality of intracerebral hemorrhage: the Tromsø Study 1995-2012. Cerebrovasc Dis Extra 2016;6:40-49.

31 Mackey J, Khoury JC, Alwell K, Moomaw CJ, Kissela BM, Flaherty ML, Adeoye O, Woo D, Ferioli S, De Los Rios La Rosa F, Martini S, Khatri P, Broderick JP, Zuccarello M, Kleindorfer D: Stable incidence but declining casefatality rates of subarachnoid hemorrhage in a population. Neurology 2016;87:2192-2197.

-32 Auer RN, Sutherland GR: Primary intracerebral hemorrhage: pathophysiology. Can J Neurol Sci 2005;32(suppl 2):S3-S12.

33 Intengan HD, Schiffrin EL: Vascular remodeling in hypertension: roles of apoptosis, inflammation, and fibrosis. Hypertension 2001;38:581-587.

-34 Eriksson M, Stegmayr B, Lundberg V: MONICA quality assessments. Scand J Public Health Suppl 2003;61: 25-30.

-35 Linnersjo A, Hammar N, Gustavsson A, Reuterwall C: Recent time trends in acute myocardial infarction in Stockholm, Sweden. Int J Cardiol 2000;76:17-21.

-36 Koster M, Asplund K, Johansson A, Stegmayr B: Refinement of Swedish administrative registers to monitor stroke events on the national level. Neuroepidemiology 2013;40:240-246. 
37 Andersson J, Johansson L, Ladenvall P, Wiklund PG, Stegmayr B, Jern C, Boman K: C-reactive protein is a determinant of first-ever stroke: prospective nested case-referent study. Cerebrovasc Dis 2009;27:544-551.

38 Wiseman S, Marlborough F, Doubal F, Webb DJ, Wardlaw J: Blood markers of coagulation, fibrinolysis, endothelial dysfunction and inflammation in lacunar stroke versus non-lacunar stroke and non-stroke: systematic review and meta-analysis. Cerebrovasc Dis 2014;37:64-75.

-39 Wiseman SJ, Doubal FN, Chappell FM, Valdés-Hernández MC, Wang X, Rumley A, Lowe GD, Dennis MS, Wardlaw JM: Plasma biomarkers of inflammation, endothelial function and hemostasis in cerebral small vessel disease. Cerebrovasc Dis 2015;40:157-164.

40 Kanaji T, Okamura T, Osaki K, Kuroiwa M, Shimoda K, Hamasaki N, Niho Y: A common genetic polymorphism (46 C to T substitution) in the $5^{\prime}$-untranslated region of the coagulation factor XII gene is associated with low translation efficiency and decrease in plasma factor XII level. Blood 1998;91:2010-2014.

-41 Gordon EM, Donaldson VH, Saito H, Su E, Ratnoff OD: Reduced titers of Hageman factor (factor XII) in Orientals. Ann Intern Med 1981;95:697-700.

-42 Law MR, Wald NJ, Wu T, Hackshaw A, Bailey A: Systematic underestimation of association between serum cholesterol concentration and ischaemic heart disease in observational studies: data from the BUPA study. BMJ 1994;308:363-366. 